\title{
EXPANSIÓN DEL MERCADO INMOBILIARIO DE VIVIENDA A PARTIR DEL PROGRAMA CIUDADES DEL BICENTENARIO
}

\section{EXPANSION OF THE REAL ESTATE HOUSING MARKET FROM THE BICENTENNIAL CITIES PROGRAM}

\author{
Francisco Javier Rosas Ferrusca ${ }^{1}$ \\ Viridiana Rodríguez Sánchez ${ }^{2}$
}

\begin{abstract}
Resumen
Uno de los principales elementos de la estructura urbana que contribuye a la expansión de las ciudades es la vivienda, que asociada al mercado inmobiliario y a las políticas de desarrollo urbano transforman constantemente el tejido de las urbes. En este contexto, la zona norte del Estado de México se ha caracterizado por un acelerado crecimiento urbano y demográfico apoyado por proyectos de desarrollo regional que hacen altamente competitivos a los municipios que conforman la región IV Cuautitlán Izcalli, destacando Huehuetoca con una significativa oferta de vivienda que durante los últimos años no logrado ser vendida a través de los esquemas crediticios tradicionales, propiciando condiciones de abandono habitacional que no corresponden con los escenarios proyectados para los próximos años. Este panorama apunta a la necesidad de replantear el modelo de crecimiento urbano basado en políticas verticales que no siempre consideran la disponibilidad de servicios básicos, infraestructura, vialidad y equipamiento para la población actual y futura.
\end{abstract}

Palabras clave: Ciudades del bicentenario, Desarrollo habitacional, Mercado inmobiliario, Conjuntos urbanos.

\begin{abstract}
One of the main elements of the urban structure that contributes to the expansion of the cities is the housing, that associated with the real estate market and urban development policies constantly transform the cities. In this context, the northern part of the State of Mexico has been characterized by a rapid demographic and urban growth supported by regional development projects that make highly competitive to the municipalities that make up the region IV Cuautitlán Izcalli, highlighting Huehuetoca with a significant supply of housing than recent years not accomplished be sold through traditional credit, schemes fostering conditions of housing abandonment which do not correspond with the scenarios projected for the coming years. This situation points to the need to rethink the model of urban growth based on vertical policies that do not always consider the availability of basic services, infrastructure, transportation and equipment for the current and future population.
\end{abstract}

Keywords: bicentennial cities, housing market, housing development, urban cities.

\footnotetext{
1 Coordinador de la Unidad Académica Profesional Huehuetoca, Universidad Autónoma del Estado de México, Doctor en Administración Pública por el Instituto Nacional de Administración Pública, A. C., Maestro en Proyectos de Desarrollo Urbano por la Universidad Iberoamericana. Miembro del Cuerpo Académico Planeación, Urbanismo y Medio Ambiente. Perfil PROMEP. E-mail: ferrusca2001@yahoo.com.mx

${ }^{2}$ Licenciada en Planeación Territorial y estudiante de la Maestría en Estudios de la Ciudad (CONACyT) de la Facultad de Planeación Urbana y Regional, Universidad Autónoma del Estado de México. E-mail: viris_rodsan@live.com.mx
} 
"La opinión en torno a la vivienda se generaliza al decir que todo es cuestión de saber cuánto cuesta construirla y cómo se puede vender. Sin embargo, dentro de la expansión urbana la construcción de vivienda nueva siempre ha sido el vehículo del crecimiento de la mancha urbana, y la planeación ha fungido como legitimadora de esta ciudad extensa."

Francisco J. Fragoso Becerril (2014).

\section{INTRODUCCIÓN}

La participación del Estado en la producción de vivienda en el contexto mexicano se ha modificado desde 1992 con la reforma al Instituto del Fondo Nacional de la Vivienda para los Trabajadores (INFONAVIT), que permitía el abandono de las actividades de construcción por parte de éste (Aguilar, 2010), situación que dio paso de acuerdo con Schteigart y García (2006), a un mercado abierto que favorecía una relación directa entre desarrolladores inmobiliarios y derechohabientes. Sin la intervención directa del Estado en la construcción de vivienda los desarrolladores inmobiliarios plasmaron "un nuevo tipo de expansión territorial con formas de ocupación de suelo difusas y en general nuevas formas de consumirlo abarcando mucho territorio" (Rodríguez, 2006: 22); en este sentido la política habitacional en México se ha caracterizado por una producción masiva de vivienda bajo la figura legal de conjuntos urbanos localizados cada vez a mayor distancia de las ciudades centrales (Pedrotti, 2013), afectando la calidad de vida de la población.

De acuerdo con Pedrotti (2013), en el Estado de México para el periodo 1999-2004 se autorizaron y construyeron 63 mil viviendas, cifra comparable con la producción de los estados de Aguascalientes, Hidalgo, Guanajuato, Yucatán, Chihuahua, Tabasco, Morelos, Zacatecas y Baja California Sur; mientras que para el periodo 2000-2010, la producción de viviendas aumentó a 617,250 distribuidas en 325 conjuntos urbanos (SEDU, citado por Pedrotti, 2013).

De esta producción, el municipio de Huehuetoca, ubicado al norte del Estado de México participa con 67,592 viviendas autorizadas en un periodo de diez años. La situación de la vivienda guarda importantes retos para la administración municipal, ya que la infraestructura hidráulica y el equipamiento productivo se encuentran rebasados por las necesidades de la población.

En este contexto, el objetivo del presente artículo consiste en analizar la expansión del mercado inmobiliario de vivienda a partir del Programa de Desarrollo Urbano Ciudades del Bicentenario, el cual tiene como una de sus seis sedes al municipio de Huehuetoca, Estado de México. Tomando en cuenta los objetivos planteados y los alcances dirigidos al municipio; los 
resultados de este análisis permiten por un lado, identificar la acelerada evolución del área urbana partir de la construcción de vivienda, y por otro, la postura gubernamental a través de los principios, dimensiones e indicadores de gobernanza territorial al desarrollo urbano en Huehuetoca.

\section{ANÁLISIS DEL PROGRAMA DE DESARROLLO URBANO CIUDADES DEL BICENTENARIO}

El programa de desarrollo urbano "Ciudades del Bicentenario" fue implementado por la Secretaría de Desarrollo Urbano del Gobierno del Estado de México en octubre del 2008, para conmemorar dos años más tarde el bicentenario de la independencia de México. Este instrumento constituye una estrategia de ordenamiento territorial para reorientar el crecimiento urbano en la zona norte del Estado de México, configurando ciudades altamente competitivas. No obstante, en municipios como Huehuetoca, el umbral de crecimiento establecido por el programa se ha rebasado, situación que tiene como fundamento una decisión vertical por parte del gobierno estatal donde no se tomó en cuenta la situación coyuntural del municipio, que si bien presenta un mercado de suelo accesible, requiere de mayor equipamiento productivo e infraestructura hidráulica y de comunicaciones para atender los requerimientos de la población que habitaría estos conjuntos urbanos.

\section{ANTECEDENTES DEL PROGRAMA CIUDADES DEL BICENTENARIO}

El municipio de Huehuetoca pertenece a la Zona Metropolitana del Valle de México (ZMVM), por criterios de integración funcional. Su localización en la zona norte del Estado de México permite tener mayor conectividad con entidades de la región centro; es por ello que la potencialidad de su ubicación ha sido aprovechada por el gobierno estatal y municipal, incidiendo en el crecimiento urbano a través de instrumentos normativos que modifican los usos de suelo para dar certeza jurídica a los desarrolladores inmobiliarios. La necesidad por incrementar la zona urbana en Huehuetoca, tiene como antecedente la política que se estableció a finales de 1950 en el Distrito Federal, cuyo objetivo consistía en limitar el crecimiento de la zona industrial y de los conjuntos urbanos (GEM, 2005). Posteriormente en el año 2000, el gobierno del Distrito Federal publicó el "Bando Número Dos", el cual constituye un decreto por el que se restringe el crecimiento de unidades habitacionales y desarrollos comerciales en nueve delegaciones: Álvaro Obregón, Coyoacán, Cuajimalpa de Morelos, Iztapalapa, Magdalena Contreras, Milpa Alta, Tláhuac, Tlalpan y Xochimilco, con la finalidad de revertir el crecimiento desordenado de la ciudad (JGDF, 2000). 
Derivado de las restricciones aprobadas por el gobierno del Distrito Federal, el gobierno del Estado de México y el H. Ayuntamiento de Huehuetoca, tomaron en cuenta la participación de la población de las comunidades de Barrio San Bartolo, Fraccionamiento Casa Nueva, Barrio de Salitrillo, Barrio La Cañada, Barrio San Pedro Xalpa, Barrio Santiago Tlaltepoxco, Barrio Santa María y Fraccionamiento Ex Hacienda de Xalpa. En el año 2002, publican el Plan Parcial de Desarrollo Urbano de Santa Teresa, con el propósito de incorporar 1,039.20 hectáreas al área urbana actual para la conformación de equipamiento y distritos habitacionales, satisfaciendo las necesidades de vivienda que manifestaba la población. (GEM, 2002).

De acuerdo con el Plan Parcial de Desarrollo Urbano de Santa Teresa (2002), el área fue seleccionada por su potencialidad para constituir un polo de desarrollo receptor del crecimiento urbano alterno a los municipios consolidados que integran la región del Valle Cuautitlán - Pachuca y por tanto pertenecen a la ZMVM. Adicionalmente, el área de Santa Teresa permitiría el mejoramiento de la distribución de la población y el impulso de la industria existente en la zona.

Tres años más tarde en junio del 2005, el Gobierno del Estado de México, publicó el Plan Regional de Desarrollo Urbano del Valle Cuautitlán-Texcocoso , el cual enfatiza la localización de los municipios que integran la región Cuautitlán-Texcoco, como un factor determinante para su desarrollo económico por medio de la conectividad con estados de la región centro como Hidalgo, Tlaxcala, Puebla, Morelos y el Distrito Federal; además de un vínculo estratégico con el Golfo de México, el Bajío y el Océano Pacífico (GEM, 2005).

La región presenta un aumento progresivo de la urbanización que sigue una tendencia desarticulada, por ello el gobierno estatal planteó como estrategia de crecimiento urbano la instrumentación de políticas de control, crecimiento y consolidación; para el caso del municipio de Huehuetoca se aplica una política de consolidación con el objetivo de "orientar, confinar y apoyar el desarrollo urbano y la densificación de las áreas localizadas a lo largo del corredor Huehuetoca Los Reyes Acozac" (GEM, 2005: 135), para lo cual es de suma importancia la articulación de actividades industriales y de servicios que sustenten la dinámica funcional del municipio hacia la ZMVM.

De acuerdo con el Plan Regional del Valle Cuautitlán-Texcoco (2005), la consolidación del crecimiento urbano en el municipio de Huehuetoca debe sustentarse en la mezcla de usos de suelo, el fortalecimiento del corredor ligado al Tratado de Libre Comercio (TLC) con la finalidad de vincular a la pequeña y mediana industria con el mercado externo; y la implementación de servicios de transporte masivo (tren suburbano, proyecto inconcluso desde el año 2000) que

\footnotetext{
${ }^{3}$ Para fines del ordenamiento sectorial del territorio el municipio de Huehuetoca, pertenece a esta región, integrada además por los municipios de Coyotepec, Cuautitlán Izcalli, Tepotzotlán y Villa del Carbón.
} 
permitan el desplazamiento de la población desde su lugar de residencia a las áreas de trabajo, escuela y ocio. Este instrumento normativo de carácter estatal justifica la modificación parcial al Plan Municipal de Desarrollo Urbano de Huehuetoca, publicada el 26 de julio del 2005, bajo iniciativa de la empresa residencial Atizapán S. A. de C.V., aprobada en la administración municipal 2003-2006 por el H. Ayuntamiento de Huehuetoca, el objetivo de esta modificación consistió en incorporar al desarrollo urbano el sector conocido como La Teña, ${ }^{4}$ entre las estrategias de esta modificación se establece la necesidad de permitir altas densidades en las viviendas, la incorporación de 47,080 personas al crecimiento urbano del municipio en un lapso de ocho años posteriores a la modificación del plan; además de la mezcla de usos de suelo permitiendo la descentralización de servicios y el crecimiento urbano ordenado (H. Ayuntamiento de Huehuetoca, 2005).

Para el año 2008, el Programa Estatal de Desarrollo Urbano plantea como objetivo incidir en la orientación del crecimiento urbano de los municipios localizados al norte del Estado de México que integran la ZMVM; la estructuración de éstos se basa en la constitución de ejes y enlaces para detonar y equilibrar el desarrollo; este programa asigna al municipio de Huehuetoca el papel de centro de población estructurador del ordenamiento territorial (GEM, 2008).

La revisión de los instrumentos de planeación en el ámbito estatal y municipal permiten ubicar al municipio de Huehuetoca con un importante papel como sede del crecimiento urbano; el cual para llevarse a cabo contemplaba los cambios en el uso de suelo favoreciendo la construcción de vivienda, la necesidad de equipamiento, infraestructura y servicios de transporte masivo que sumados al impulso y consolidación de la industria harían de Huehuetoca uno de los municipios más importantes de la ZMVM. Estos instrumentos son la base para la formulación del programa de desarrollo urbano Ciudades del Bicentenario.

\section{DEFINICIÓN DEL PROgRAMA DE DESARROLlo URBANO CIUdAdES DEL BICENTENARIO}

El programa de Desarrollo Urbano Ciudades del Bicentenario, pretendía seguir una visión de gobernanza ${ }^{5}$ por medio de un consenso entre los distintos actores, para el logro de proyectos de inversión; sin embargo, su diseño obedeció a una formulación vertical de dos actores: el gobierno estatal a través de la Secretaría de Desarrollo Urbano, y el sector empresarial

\footnotetext{
${ }^{4}$ Además de Huehuetoca cabecera municipal, comprende las comunidades de La Cañada, Santa Teresa, Jorobas, Salitrillo, Casa Nueva, San Bartolo y Santa María.

${ }^{5}$ De acuerdo con el documento de la Comisión 3 en el 2009, el Estado de México incursiona en el modelo de gobernanza urbana integrada a través de una estrategia de ordenamiento territorial denominada Ciudades del Bicentenario.
} 
representado por la Comisión Coordinadora para el Impulso a la Competitividad en el Estado de México ${ }^{6}$, a pesar de que el programa se implementó en seis municipios los gobiernos locales y la sociedad civil de éstos, no participaron en su elaboración, implementación y desarrollo. El programa Ciudades del Bicentenario constituye una estrategia de ordenamiento territorial basada en el modelo de ciudad dispersa ${ }^{7}$, con polos de desarrollo ubicados al norte de la ZMVM en los municipios de Atlacomulco, Almoloya de Juárez, Jilotepec, Huehuetoca, Tecámac y Zumpango; planteando un total de 28,597 hectáreas a urbanizar, alcanzando su potencial de crecimiento ordenado en el año 2020 (GEM b, 2008). Estos municipios se interconectarían mediante el Arco Norte ${ }^{8}$, que además permite la integración con estados de la región centro y cuatro puertos al este Tuxpan y Veracruz, al oeste Manzanillo y Lázaro Cárdenas (Comisión Coordinadora para el Impulso a la Competitividad en el Estado de México, 2007).

\footnotetext{
${ }^{6}$ La participación del sector privado en la comisión está constituida por: el Consejo Coordinador Empresarial (CCE), Asociación de Industriales del Estado de México (AlIEM), Consejo Empresarial del Valle de Toluca (CEVAT), Consejo Patronal de la República Mexicana (COPARMEX) Estado de México, Centro Empresarial Metropolitano (COPARMEX), Cámara Nacional de la Industria de la Transformación (CANACINTRA) Estado de México, Cámara Nacional de Comercio (CANACO Toluca), Consejo Mexicano de Comercio Exterior, Inversión y Tecnología (COMCE) Estado de México, Fundación México Estados Unidos para la Ciencia- Metrópolis 2025, Consejo Ejecutivo de Empresas Globales, Asociación Mexicana de Parques Industriales Privados, Confederación de Cámaras Nacionales de Comercio, Servicios y Turismo del Estado de México, Cámara Nacional de Autopartes (GEM, 2007).

${ }^{7}$ Este patrón es coherente con el fenómeno del urban sprawl norteaméricano o dispersión urna producida por la des-densificación de las ciudades sin un orden o criterio urbanístico claro y predefinido, existe una carencia de límites entre lo urbano y lo rural; equivale a la concepción de ciudad como territorio en expansión sin límites (Pedrotti, 2013: 5).

${ }^{8}$ El Arco Norte es uno de los más importantes proyectos carreteros del gobierno federal, se ubica en los estados de México, Hidalgo, Tlaxcala y Puebla, librando la zona metropolitana de la Ciudad de México. Cruza importantes Ejes Carreteros como México - Morelia, México - Querétaro, México - Pachuca, México Tulancingo y México Puebla; mejorando la logística de transporte, ya que une la región sureste de la República con el norte y noreste del país. Inicia operación en marzo del 2006 con 18.5 km en el entronque México a Querétaro -Tula, la construcción de esta autopista terminó en el año 2011 con un total de 223 km.
} 
Figura 1: Localización de las Ciudades del Bicentenario

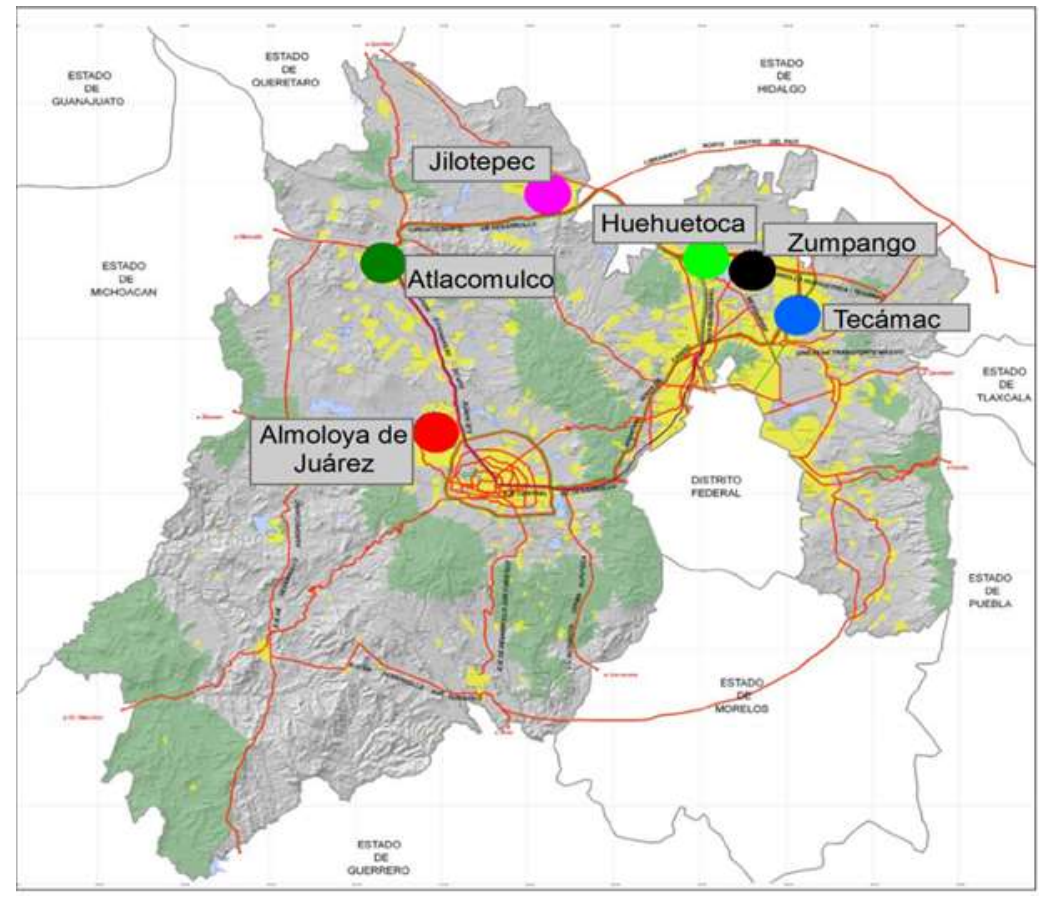

Fuente: Gobierno del Estado de México, 2008.

Los sustentos para la integración de las Ciudades del Bicentenario de acuerdo con la Comisión Coordinadora para el Impulso a la Competitividad en el Estado de México (2007: 1) son: "Estructurar y ordenar el territorio para generar ciudades competitivas y regiones de desarrollo, orientando el crecimiento a las zonas más aptas para usos urbanos; promover la infraestructura estratégica de la entidad; y estimular y orientar inversiones para crear las condiciones físicas que permitan el desarrollo equilibrado de actividades productivas y satisfactores sociales".

Con base en este planteamiento, las Ciudades del Bicentenario se definen como:

Ciudades modelo, autosuficientes, debidamente estructuradas, sustentables y altamente competitivas (...) serán ciudades competitivas y equitativas, industriales, vanguardistas y tecnológicas en un medio ambiente natural protegido (...) Ofrecen todo su potencial, recursos humanos, naturaleza e infraestructura, mano de obra calificada, amplia oferta educativa, certidumbre legal para el uso y ocupación del suelo transparencia en trámites y autorizaciones y una adecuada planeación para el desarrollo sustentable. (SEDU, 2008: 8-9).

De acuerdo con la Comisión Coordinadora para el Impulso a la Competitividad en el Estado de México (2007), la puesta en marcha de las Ciudades del Bicentenario debía contar con apoyo institucional de los tres órdenes de gobierno en materia de programación y presupuestación para la ejecución de proyectos estratégicos, así como del sector privado en la inversión productiva. Por 
ello, el objetivo del programa consiste en: "orientar y redistribuir el crecimiento urbano de la entidad hacia municipios que presentan condiciones apropiadas para recibir impulso con una visión de futuro a largo plazo, incluyente, consensuada y socialmente participativa" (SEDU, 2008: 5). Las Ciudades del Bicentenario se eligieron de acuerdo con el gobierno del Estado de México (2008 b), por su ubicación, capacidad para recibir incrementos poblacionales significativos, capacidad para alojar infraestructura y equipamientos estratégicos; además de la disponibilidad de vías de comunicación suficientes que permiten su articulación regional estatal e inclusive nacional. Aunado a esto, con apoyo del Consejo Consultivo para la Competitividad del Estado de México, se definió el perfil de cada ciudad:

\section{Cuadro 1. Perfil de las Ciudades del Bicentenario}

\begin{tabular}{ll}
\hline Ciudad & Perfil \\
\hline Almoloya de Juárez & Centro regional del conocimiento y la tecnología \\
\hline Atlacomulco & Centro agroindustrial y biotecnológico \\
Jilotepec & Centro de innovación para las ciencias y el \\
& entretenimiento \\
Huehuetoca & Centro de logística y distribución regional \\
Zumpango & Centro comercial y de servicios, ecoturístico y acuífero \\
Tecámac & Tecnópolis y parques industriales de alta tecnología \\
\hline
\end{tabular}

Fuente: Secretaría de Desarrollo Urbano, 2008.

De acuerdo con el gobierno del Estado de México (2008 b), para el caso de los municipios de Jilotepec, Huehuetoca, Tecámac y Zumpango se tomó en cuenta la existencia de importantes vialidades como la autopista México- Querétaro y la México-Pachuca, además del enlace con el Golfo de México realizado mediante el Arco Norte.

\section{LINEAMIENTOS GENERALES DEL PROGRAMA}

Los lineamientos que permitieron la implementación del programa Ciudades del Bicentenario, de acuerdo con la Dirección de Planeación Urbana (2008), son:

1. Destinar amplias superficies para la creación de espacios públicos, áreas verdes y recreativas, brindando sustentabilidad ambiental;

2. Orientar el desarrollo de las ciudades vinculando las zonas urbanas actuales con las áreas de nuevo crecimiento;

3. Canalizar y gestionar recursos para la construcción de infraestructura de cabecera, la creación de equipamientos regionales y el mejoramiento urbano;

4. Buscar la integración plena en términos de conectividad vial y de transporte; 
5. Promover la diversidad en la tipología y oferta de vivienda; y

6. Privilegiar la óptima utilización del espacio urbano y la mezcla de usos de suelo, promoviendo el crecimiento vertical.

\section{LINEAMIENTOS ESPECÍFICOS PARA EL MUNICIPIO DE HUEHUETOCA}

Para el caso específico del municipio de Huehuetoca, la Comisión Coordinadora para el Impulso a la Competitividad en el Estado de México (2007), le asignó el papel de centro de logística y distribución regional basándose tanto en su ubicación como en la conectividad con Querétaro y Pachuca; además, de que el territorio municipal es el espacio donde convergen las líneas de ferrocarril que van de la Ciudad de México al norte del país.

Considerando las tendencias de crecimiento urbano en el municipio, se determinaron los siguientes lineamientos (GEM, 2008 b):

1. Consolidación de usos industriales y de alta densidad con mezcla de actividades industriales, comercio y servicios;

2. Vocación para constituirse en el más importante centro logístico del norte de la ZMVM. Generación de empleo con importantes inversiones en industria;

3. Aprovechamiento de vialidades regionales, específicamente la carretera Jorobas-Tula, y el Circuito Exterior Mexiquense;

4. Saneamiento del Río Cuautitlán;

5. Estructuración urbana con base en distritos, colonias, y barrios con centros y corredores urbanos. 
Figura 2. Proyecto "Ciudad Bicentenario Huehuetoca"

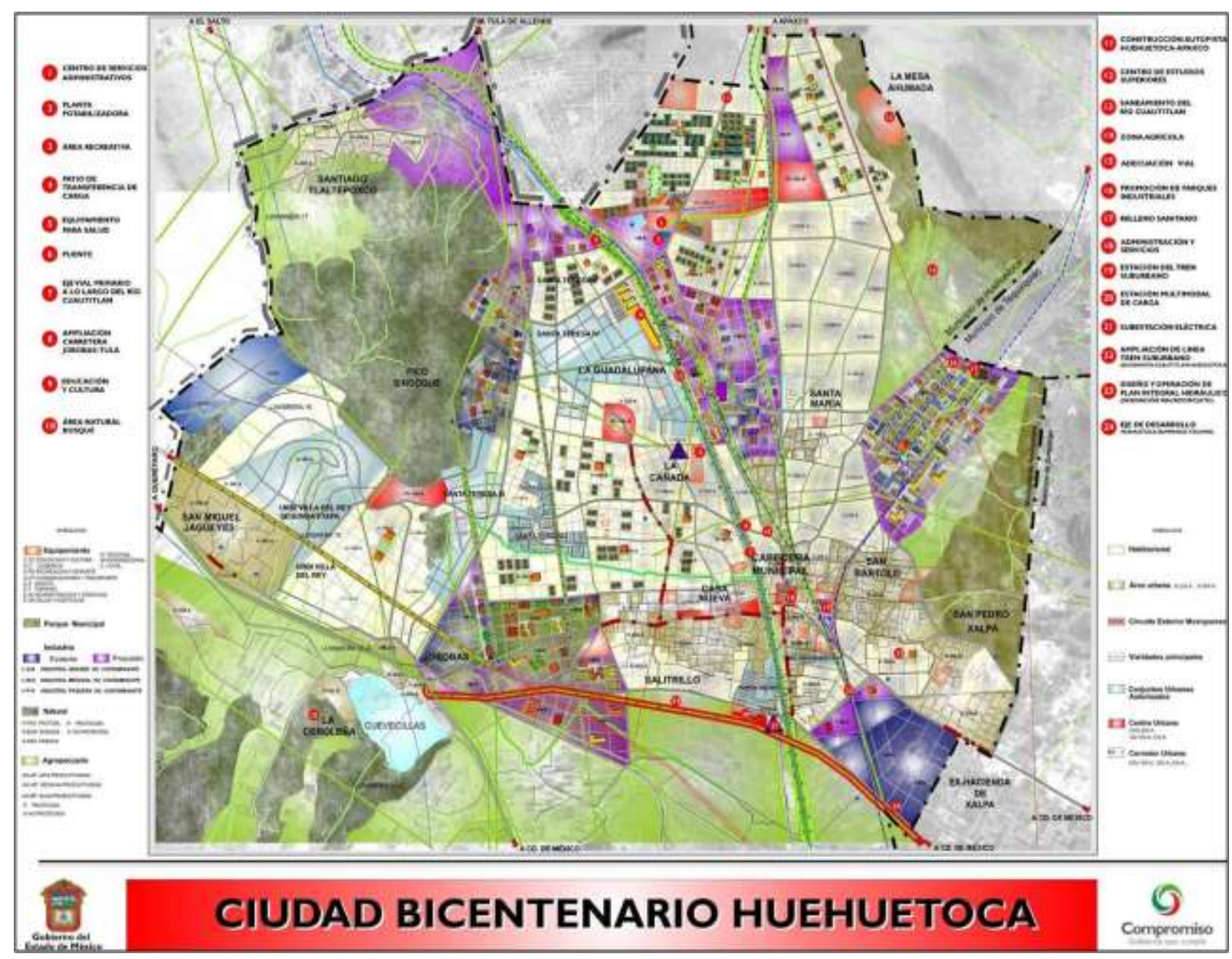

Fuente: Gobierno del Estado de México b, 2008.

INSTRUMENTACIÓN DEL PROGRAMA

Si bien el diseño del programa Ciudades del Bicentenario siguió una visión vertical, para su instrumentación se pretendía aplicar una dimensión horizontal aludiendo a las relaciones partenariales entre actores públicos, privados y la sociedad civil, involucrándose de la siguiente forma (ver cuadro 2). 
Cuadro 2. Formas de participación de los actores públicos, privados y sociales en programa Ciudades del Bicentenario

\begin{tabular}{|c|c|c|}
\hline Actores públicos & $\begin{array}{l}\text { Actores privados/sector } \\
\text { financiero }\end{array}$ & Actores sociales \\
\hline $\begin{array}{l}\text { Decisión política y el conjunto } \\
\text { de instrumentos para orientar } \\
\text { la inversión privada hacia las } \\
\text { zonas seleccionadas. }\end{array}$ & $\begin{array}{l}\text { Brindar recursos para el } \\
\text { desarrollo de la región, de la } \\
\text { infraestructura y equipamiento } \\
\text { regional. }\end{array}$ & $\begin{array}{l}\text { Enriquecer proyectos a través } \\
\text { de opiniones y sugerencias, } \\
\text { emitidas en la consulta pública } \\
\text { mismas que serán útiles para } \\
\text { aprobar los Planes de } \\
\text { Desarrollo Urbano. }\end{array}$ \\
\hline $\begin{array}{l}\text { Dirigir la inversión pública y } \\
\text { privada crear } \\
\text { infraestructura de cabecera. }\end{array}$ & $\begin{array}{l}\text { Adquirir suelo, construyendo } \\
\text { en la ciudad, vivienda, } \\
\text { industria, comercio y servicios. }\end{array}$ & $\begin{array}{l}\text { Instalación de consejos } \\
\text { consultivos municipales para } \\
\text { vigilar el desarrollo de los } \\
\text { planes y proyectos de las } \\
\text { Ciudades Bicentenario. }\end{array}$ \\
\hline $\begin{array}{l}\text { Orientar programas de las } \\
\text { dependencias estatales hacia } \\
\text { las Ciudades Bicentenario } \\
\text { acorde a los planes de } \\
\text { desarrollo urbano que } \\
\text { sustentarán los proyectos a } \\
\text { través de la emisión de } \\
\text { autorizaciones y licencias. }\end{array}$ & $\begin{array}{l}\text { Recursos crediticios necesarios } \\
\text { para la construcción de la } \\
\text { nueva ciudad. }\end{array}$ & \\
\hline
\end{tabular}

Fuente: Elaboración propia con base en el Gobierno del Estado de México b 2008.

Como se muestra en el cuadro 2, la participación de los actores públicos se basa en orientar, dirigir e instrumentar el programa Ciudades del Bicentenario, los recursos económicos serían aportados por los actores privados, a los cuales se les permitiría la adquisición de suelo con fines de desarrollo económico, mientras que la participación de la sociedad se limita a opinar sobre lo ya planteado. No obstante, en la práctica la participación de los actores sociales sólo se concretó a la conformación de los Consejos Consultivos Municipales, figura que únicamente otorgaría su opinión sobre proyectos planteados por los inversionistas que se esperaba trajera resultados positivos para todo el municipio. En esta lógica cabe destacar la participación de la Secretaría de Desarrollo Urbano para la determinación de políticas específicas que permitieran la autorización de nuevos conjuntos urbanos de carácter habitacional, atendiendo la demanda que genera el crecimiento económico, la capacidad de infraestructura y servicios (Poder ejecutivo del estado, 2008). 


\section{ALCANCES DEL PROGRAMA DIRIGIDOS AL MUNICIPIO DE HUEHUETOCA}

Con base en los lineamientos del programa Ciudades del Bicentenario previamente descritos, la Secretaría de Desarrollo Urbano (2008), centró los objetivos del programa en cuatro variables: incremento poblacional, demanda de vivienda, clasificación del territorio y generación de empleo, elaborando escenarios con un corte temporal del 2008 al 2020 para las seis ciudades, como se muestra a continuación.

Cuadro 3. Escenarios previstos por el programa Ciudades del Bicentenario, 2008-2020

\begin{tabular}{|c|c|c|c|c|}
\hline \multirow[t]{2}{*}{ Población 2005} & \multicolumn{3}{|c|}{ Incremento poblacional } & \multirow[t]{2}{*}{ Población 2020} \\
\hline & $2008-2011$ & 2011-2015 & $2015-2020$ & \\
\hline 59,721 & 55,044 & 106,159 & 190,195 & 411,119 \\
\hline \multirow[t]{2}{*}{ Vivienda 2005} & \multicolumn{3}{|c|}{ Demanda de vivienda } & \multirow[t]{2}{*}{ Vivienda 2020} \\
\hline & 2008-2011 & 2011-2015 & $2015-2020$ & \\
\hline 13,192 & 12,861 & 24,804 & 44,438 & 95,295 \\
\hline \multirow{2}{*}{$\begin{array}{l}\text { Superficie total } \\
\text { municipal }\end{array}$} & \multicolumn{3}{|c|}{ Superficie urbana } & \multirow{2}{*}{$\begin{array}{l}\text { Potencial de } \\
\text { empleo sector } \\
\text { secundario }\end{array}$} \\
\hline & Actual & Urbanizable & Ambas & \\
\hline 16,920 & 2,130 & 3,192 & 5,322 & 109,725 \\
\hline
\end{tabular}

Fuente: Elaboración propia con base en la Dirección Operativa de Desarrollo Urbano, 2008.

Los escenarios planteados en el cuadro 3 toman como base la población del año 2005, en tres cortes de tiempo, el primero de 2008-2011 de tres años, estimando un incremento poblacional de 55,044 habitantes con una demanda de vivienda de 12,861; el segundo corte es del 2011-2015 de cuatro años con un incremento poblacional de 106,159 habitantes, y una demanda de vivienda de 24,804. El tercer corte de tiempo tiene una duración de cinco años del 2015 al 2020 y estima un incremento demográfico de 190,195 personas, con requerimientos de 44,438 viviendas.

Desde esta perspectiva, para el año 2020 se tendría una población de 411,119 personas y un total de viviendas de 95,295. Es conveniente destacar, que ningún documento del Gobierno del Estado de México describe los métodos para realizar las proyecciones, ni tampoco porque los tres cortes de tiempo no mantienen un mismo periodo de años. Con referencia a la superficie urbana, la Secretaría de Desarrollo Urbano previó un área urbanizable de 3,192 hectáreas de las cuales: 1,308 estarían destinadas a uso habitacional, 861 a industria, 63 para equipamiento, 478 para áreas verdes y 482 a vialidades. La oferta laboral en el sector secundario se garantizaría por la llegada de más industria generando empleo para 109,725 personas al año 2020. 
Estas cuatro variables desde la perspectiva de los actores estatales y privados garantizarían la competitividad de la Ciudad Bicentenario Huehuetoca; sin embargo, a cuatro años de la puesta en marcha de este programa por parte del Ayuntamiento, se han obtenido resultados diferentes a los previstos, sin que exista una revisión, reajuste y/o evaluación de los lineamientos por parte de ningún actor que permita la optimización y mejoramiento del programa.

La falta de conocimiento de la problemática territorial que el municipio tenía previo a la implementación del programa, aunado a una visión vertical en el diseño de éste, donde no se involucró al gobierno local ni a la sociedad civil, generan como consecuencia afectaciones enfocadas principalmente al sector vivienda, con efectos colaterales al medio ambiente y capacidad de infraestructura que cuestionan la acción del gobierno local para resolver estos retos.

\section{COMPARACIÓN DE LOS ESCENARIOS DE INCREMENTO POBLACIONAL Y DEMANDA DE VIVIENDA}

Es importante mencionar, que en los documentos consultados sobre el diseño del programa de Desarrollo Urbano Ciudades del Bicentenario, no se específica la metodología utilizada para la construcción de escenarios; por ello con la finalidad de establecer la verticalidad del programa y el desconocimiento de la dinámica municipal; se realizó una comparación de los escenarios previstos por la Secretaría de Desarrollo Urbano y las proyecciones elaboradas por el Consejo Nacional de Población (CONAPO), con un horizonte temporal al 2020. Así, para el año 2011 el CONAPO proyectó una población de 110,515 habitantes, lo que permite estimar un incremento poblacional del periodo 2008-2011 de 31,811 individuos, cifra que difiere en 23,233 personas con el escenario establecido por el programa Ciudades del Bicentenario.

El segundo corte de tiempo del 2011-2015, se calcula un incremento poblacional de 22,230 habitantes, cifra que difiere en 83,929 individuos con el escenario establecido por la Secretaría de Desarrollo Urbano; conviene destacar que al año de realización de esta investigación (2014), se calcula un incremento de 17,770 habitantes cifra que indica un sesgo importante para esta comparación. En esta misma lógica para el corte 2015-2020, se proyecta un incremento poblacional de 16,881 personas mientras que el incremento planteado en el programa es de 190,195, lo que refleja una diferencia de173,314 individuos.

De acuerdo con CONAPO (2014), para el año 2020 la proyección poblacional para Huehuetoca indica un total de 149,426 habitantes, si se compara con la cifra proyectada por la Secretaría de Desarrollo Urbano que es de 411,119 habitantes se tendría una diferencia de 261,693 
habitantes. La diferencia entre las proyecciones del programa Ciudades del Bicentenario y CONAPO, se visualiza en la siguiente gráfica.

\section{Gráfica 1. Comparación entre proyecciones de incremento poblacional}

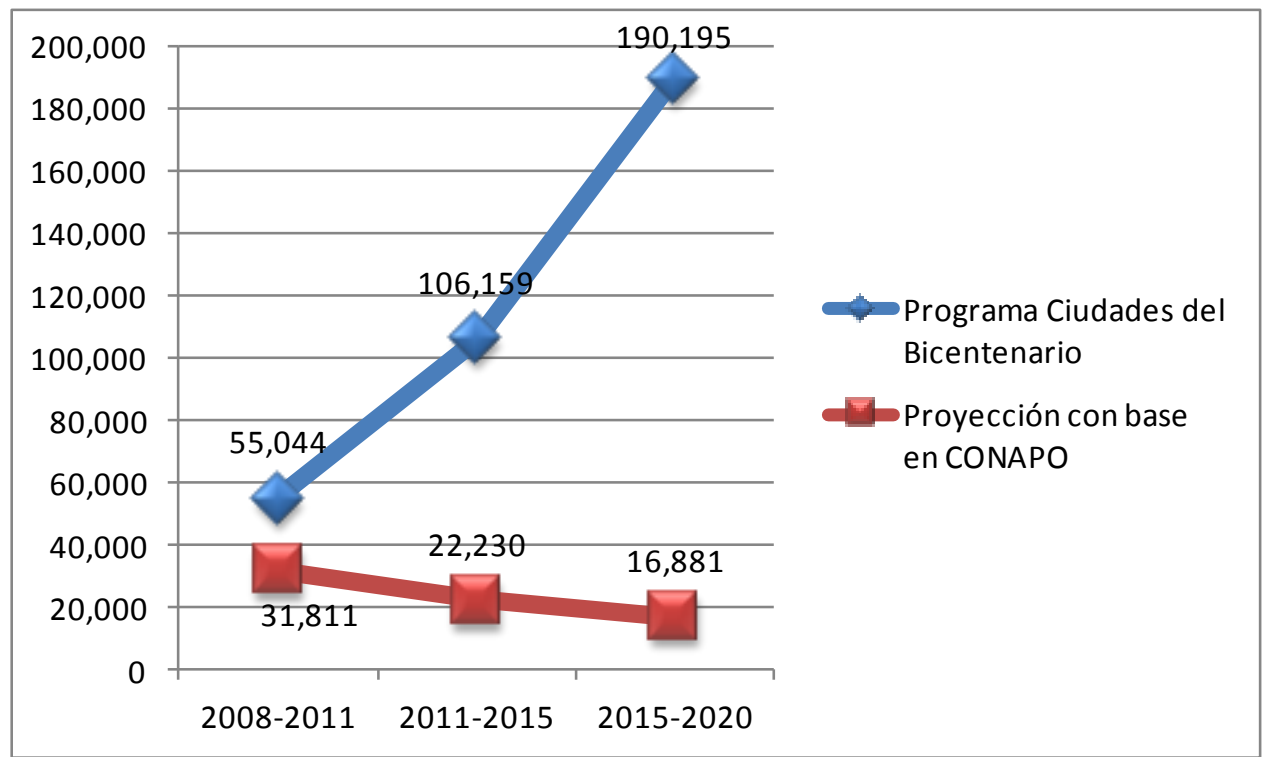

Fuente: Elaboración propia con base en la Secretaría de Desarrollo Urbano 2008 y CONAPO 2014.

En este sentido, es importante inferir que derivado de las proyecciones de población se realizaron los cálculos para la demanda de vivienda, la cual muestra un comportamiento peculiar, mismo que se explica a continuación.

\section{COMPORTAMIENTO DE LA VIVIENDA}

A partir de la puesta en marcha del programa Ciudades del Bicentenario en Huehuetoca, se han autorizado 31,661 viviendas pertenecientes a cuatro grupos inmobiliarios: Conjunto Parnelli, S. A., de C. V. Casas Beta del Centro, S. de R. L. de C. V. Consorcio de Ingeniería Integral S. A. de C. V. Y GEO Edificaciones, S. A. de C. V. (SEDU, 2014). Tomando en cuenta los periodos de tiempo establecidos por el programa, se tiene que para el corte 2008-2011 se autorizaron un total de 23,007 viviendas dirigidas a 103,576 personas en ocho conjuntos urbanos, como se muestra en el siguiente cuadro. 
Cuadro 4. Conjuntos urbanos autorizados en el periodo 2008-2011 en Huehuetoca, México

\begin{tabular}{|c|c|c|c|c|c|}
\hline ño & Conjunto urbano & Empresa desarrolladora & Tipo & $\begin{array}{l}\text { Número de } \\
\text { viviendas }\end{array}$ & $\begin{array}{l}\text { Población } \\
\text { beneficiada }\end{array}$ \\
\hline \multirow[t]{6}{*}{008} & $\begin{array}{l}\text { El Dorado } \\
\text { Huehuetoca (2a } \\
\text { etapa). }\end{array}$ & $\begin{array}{c}\text { Conjunto Parnelli, S. A., } \\
\text { de C.V. }\end{array}$ & $\begin{array}{l}\text { Interés } \\
\text { social }\end{array}$ & 1,971 & 8,869 \\
\hline & Santa Teresa VI B & $\begin{array}{c}\text { Casas Beta del Centro, S. } \\
\text { de R. L. de C. V. }\end{array}$ & $\begin{array}{l}\text { Interés } \\
\text { social }\end{array}$ & 1,075 & 4,837 \\
\hline & Santa Teresa VI & $\begin{array}{c}\text { Casas Beta del Centro, S. } \\
\text { de R. L. de C. V. }\end{array}$ & $\begin{array}{c}\text { Social } \\
\text { progresivo }\end{array}$ & 728 & 3,276 \\
\hline & $\begin{array}{l}\text { Hacienda las } \\
\text { Misiones }\end{array}$ & $\begin{array}{c}\text { GEO Edificaciones, S. A. } \\
\text { de C. V. }\end{array}$ & $\begin{array}{c}\text { Social } \\
\text { progresivo }\end{array}$ & 1,556 & 7,002 \\
\hline & $\begin{array}{c}\text { Hacienda } \\
\text { Guadalupe "La } \\
\text { Guiñada" }\end{array}$ & $\begin{array}{l}\text { Consorcio de Ingeniería } \\
\text { Integral S. A. de C. V. }\end{array}$ & $\begin{array}{l}\text { Interés } \\
\text { social }\end{array}$ & 2,490 & 11,205 \\
\hline & Santa Teresa VII & $\begin{array}{c}\text { Casas Beta del Centro, S. } \\
\text { de R. L. de C. V. }\end{array}$ & $\begin{array}{l}\text { Interés } \\
\text { social }\end{array}$ & 2,252 & 10,134 \\
\hline Total & & & & 10,072 & 45,323 \\
\hline \multirow[t]{4}{*}{009} & $\begin{array}{c}\text { Huehuetoca del } \\
\text { Maurel }\end{array}$ & $\begin{array}{c}\text { Inmobiliaria CAME, S. A. } \\
\text { de C. V. }\end{array}$ & $\begin{array}{l}\text { Habitacional } \\
\text { de interés } \\
\text { social }\end{array}$ & 258 & 1,206 \\
\hline & Privadas del Valle & $\begin{array}{c}\text { KE Desarrolladora S. A. } \\
\text { de C. V. }\end{array}$ & $\begin{array}{l}\text { Habitacional } \\
\text { de interés } \\
\text { social }\end{array}$ & 4,502 & 20,259 \\
\hline & $\begin{array}{c}\text { Galaxia } \\
\text { Huehuetoca }\end{array}$ & $\begin{array}{c}\text { Inmobiliaria DIAREL, S. A. } \\
\text { de C. V. }\end{array}$ & $\begin{array}{c}\text { Habitacional } \\
\text { de interés } \\
\text { social }\end{array}$ & 2,380 & 10,710 \\
\hline & $\begin{array}{c}\text { Hacienda las } \\
\text { Misiones ( } 2 \underline{a} \text { etapa) }\end{array}$ & $\begin{array}{c}\text { GEO Edificaciones, S. A. } \\
\text { de C. V. }\end{array}$ & $\begin{array}{c}\text { Tipo Social } \\
\text { Progresivo } \\
\text { (150 Viv.) e } \\
\text { Interés } \\
\text { Social (576 } \\
\text { Viv.). }\end{array}$ & 726 & 3,267 \\
\hline Total & & & & 7,866 & 35,442 \\
\hline \multirow[t]{2}{*}{010} & $\begin{array}{c}\text { El Dorado } \\
\text { Huehuetoca (3a } \\
\text { etapa) }\end{array}$ & $\begin{array}{c}\text { Conjunto Parnelli, S. A. } \\
\text { de C. V. }\end{array}$ & $\begin{array}{l}\text { Habitacional } \\
\text { de interés } \\
\text { social }\end{array}$ & 3,016 & 13,572 \\
\hline & $\begin{array}{c}\text { In House } \\
\text { Huehuetoca }\end{array}$ & $\begin{array}{l}\text { In House Estado de } \\
\text { México, S. A. de C. V. }\end{array}$ & $\begin{array}{c}\text { Habitacional } \\
\text { de interés } \\
\text { social }\end{array}$ & 2,053 & 9,239 \\
\hline Total & & & & 5,069 & 22,811 \\
\hline 011 & \multicolumn{5}{|c|}{ No registra autorizaciones } \\
\hline
\end{tabular}

Fuente: Elaboración propia con base en la Secretaría de Desarrollo Urbano, 2014. 
Como es posible apreciar en el cuadro superior, el mayor número de conjuntos urbanos fue autorizado en el año 2008 con 10,072 viviendas a cuatro desarrolladoras inmobiliarias: Conjunto Parnelli, S. A., de C. V. Casas Beta del Centro, S. de R. L. de C. V. Consorcio de Ingeniería Integral S. A. de C. V. y GEO Edificaciones, S. A. de C. V. dirigidas a una población de 45,323 personas.

Es conveniente mostrar, que para el año 2010 de acuerdo con datos de INEGI, se registran siete conjuntos urbanos: Ciudad Integral Huehuetoca (Hacienda Guadalupe La Guiñada), el Dorado Huehuetoca, Colonia Santa Teresa, Villa Urbi del Rey, Galaxia Huehuetoca, Huehuetoca del Maurel y Privadas del Valle con un total de 34,279 viviendas, de las cuales sólo 11,841 se encuentran actualmente habitadas ${ }^{9}$.

El dato global de vivienda para el periodo 2008-2011 según el programa Ciudades del Bicentenario es de 12,861 de acuerdo con lo descrito en el cuadro 4, se tiene un total de viviendas autorizadas de 23,007, cifra que rebasa por 10,146 el escenario previsto por el programa para este periodo. Ahora bien, si se toma en cuenta que el número de ocupantes por vivienda ha disminuido de 4.74 en el año 2000 a 3.71 en el 2010; los requerimientos de vivienda para el periodo 20082011 se estiman en $7,993^{10}$, cifra inferior al dato previsto y a las estadísticas de la Secretaría de Desarrollo Urbano.

Con referencia al periodo 2011-2015, la estadística de conjuntos urbanos establece un total de 8,654 viviendas en tres conjuntos urbanos: Conjunto Parnelli, S. A. de C. V., Consorcio de Ingeniería Integral S. A. de C. V. y las Inmobiliarias Sazmsacj, S. A. de C. V., Quiero Casa, S. A. de C. V., con la colaboración de CC. Moisés Abadi Duek. Los beneficios de estos conjuntos se dirigen a 33,183 personas, el cuadro cinco describe a detalle esta información.

\footnotetext{
${ }^{9}$ Es preciso señalar que los datos de INEGI no especifican cifras sobre los conjuntos urbanos restantes, ni dividen a éstos por etapas como la Secretaría de Desarrollo Urbano.

${ }^{10}$ Cifra estimada por cálculos propios mediante la fórmula Nv=Pf-Pi/Promedio de ocupantes por vivienda.
} 


\section{Cuadro 5. Conjuntos urbanos autorizados en el periodo 2011-2015 en Huehuetoca, México}

\begin{tabular}{|c|c|c|c|c|c|}
\hline Año & $\begin{array}{l}\text { Conjunto } \\
\text { Urbano }\end{array}$ & $\begin{array}{c}\text { Empresa } \\
\text { desarrolladora }\end{array}$ & Tipo & $\begin{array}{l}\text { Número de } \\
\text { Viviendas }\end{array}$ & $\begin{array}{l}\text { Población } \\
\text { beneficiada }\end{array}$ \\
\hline 2012 & $\begin{array}{c}\text { El Dorado } \\
\text { Huehuetoca } \\
\text { (4a etapa, } \\
\text { sección A y B) }\end{array}$ & $\begin{array}{c}\text { Conjunto } \\
\text { Parnelli, S. A. } \\
\text { de C. V. }\end{array}$ & $\begin{array}{c}\text { Habitacional } \\
\text { de interés } \\
\text { social }\end{array}$ & 1,280 & 5,760 \\
\hline 2013 & \multicolumn{5}{|c|}{ No registra autorizaciones } \\
\hline \multirow[t]{3}{*}{2014} & $\begin{array}{c}\text { Citara Ciudad } \\
\text { Integral } \\
\text { Huehuetoca, } \\
3^{\circ} \text { etapa, } \\
\text { Europa }\end{array}$ & $\begin{array}{c}\text { Consorcio de } \\
\text { Ingeniería } \\
\text { Integral S. A. } \\
\text { de C. V. }\end{array}$ & Interés social & 3,552 & 15,984 \\
\hline & $\begin{array}{c}\text { El Dorado } \\
\text { Huehuetoca } \\
5^{\circ} \text { etapa } \\
\text { Fracción A, B, } \\
\text { C y D }\end{array}$ & $\begin{array}{c}\text { Conjunto } \\
\text { Parnelli, S. A. } \\
\text { de C. V. }\end{array}$ & Interés social & 3,104 & 13,968 \\
\hline & $\begin{array}{l}\text { Quiero Casa } \\
\text { Huehuetoca }\end{array}$ & $\begin{array}{c}\text { Inmobiliaria } \\
\text { Sazmsacj. S. A. } \\
\text { de C. V. e } \\
\text { Inmobiliaria } \\
\text { Quiero Casa, } \\
\text { S. A. de C. V. y } \\
\text { los CC. Moisés } \\
\text { Abadi Duek }\end{array}$ & Interés social & 718 & 3,231 \\
\hline Total & & & & 7,374 & 33,183 \\
\hline
\end{tabular}

Fuente: Elaboración propia con base en la Secretaría de Desarrollo Urbano, 2014.

La información del cuadro superior destaca que el año $2014^{11}$ registra un total de 7,374 viviendas, proyectadas para una población de 33,183 personas; hasta el mes de mayo del 2014, las 24,804 viviendas propuestas para el corte 2011-2015 no se han cumplido; lo cual es positivo, ya

${ }^{11}$ El corte se realizó al mes de febrero del 2014, y es el último dato que registra la Secretaría de Desarrollo Urbano. 
que tomando en cuenta las proyecciones de población de CONAPO (2014), y el promedio de ocupantes por vivienda se calcula una demanda de 22,230 viviendas; por tanto de cumplirse el escenario del programa Ciudades del Bicentenario se superaría la demanda por 2,574 viviendas. Con referencia al periodo 2015-2020, el programa Ciudades del Bicentenario proyecta una demanda de 44,438 viviendas; sin embargo, continuando con la lógica que se ha estimado en los párrafos precedentes el requerimiento de vivienda sería de 3,898, cifra inferior en 40,540. Para el año 2020, el programa plantea un total de viviendas de 95,29512; no obstante, derivado de las tendencias tanto en el comportamiento demográfico como en el promedio de ocupantes por vivienda se proyecta una demanda de 22,538 viviendas.

La gráfica 2 muestra la comparación entre las estimaciones realizadas con base en el CONAPO y las que estable el programa Ciudades del Bicentenario.

\section{Gráfica 2. Comparación entre escenarios de vivienda}

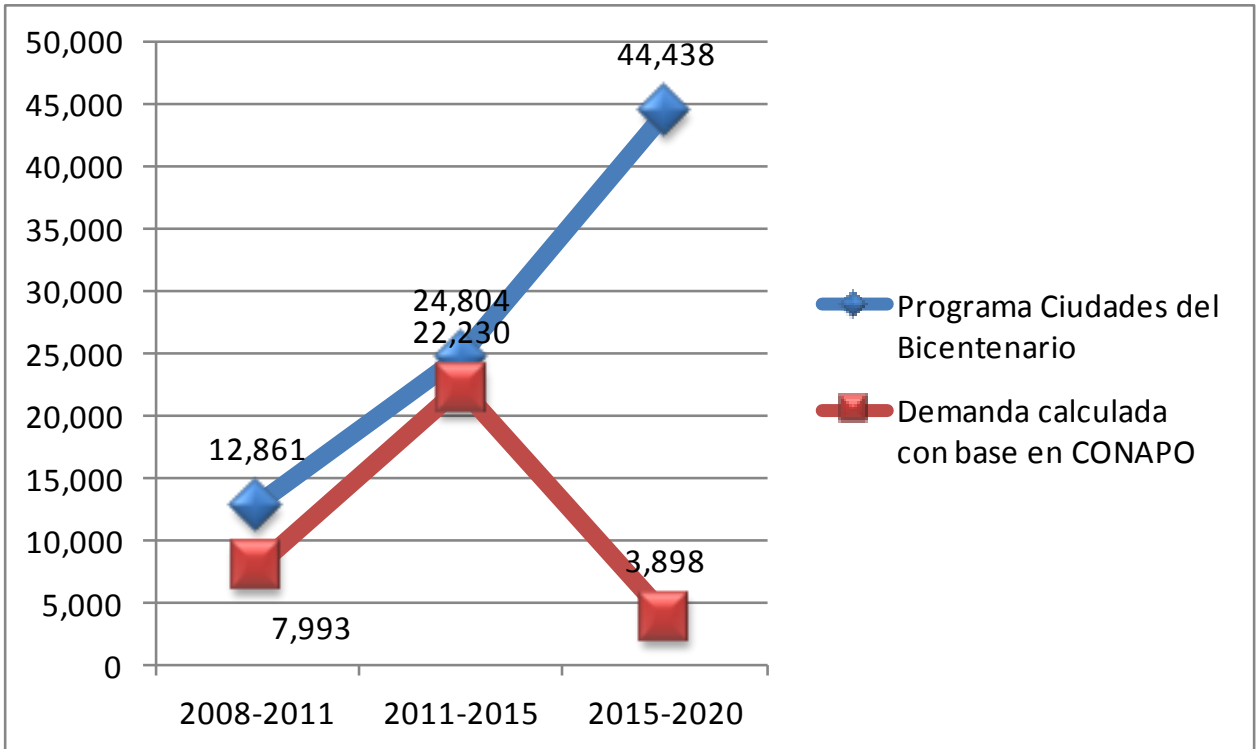

Fuente: Elaboración propia con base en la Secretaría de Desarrollo Urbano 2008 y CONAPO 2014.

Es importante tomar en cuenta que el $35.36 \%$ de los conjuntos urbanos registrados por INEGI en Huehuetoca se encuentran abandonados, esta situación permite cuestionar los alcances del programa Ciudades del Bicentenario, ya que las variables de población y vivienda se implementaron de forma aislada sin el seguimiento de proyectos como el de incentivar la actividad industrial, servicios de transporte masivo (tren suburbano) y el saneamiento del Río Cuautitlán,

\footnotetext{
${ }^{12}$ La proyección final de vivienda se realizó para el periodo 2008-2020.
} 
factores que influyen directamente en la calidad de vida de la población, y que a su vez han incidido en el abandono habitacional.

\section{INCIDENCIA DEL PROGRAMA CIUDADES DEL BICENTENARIO EN LA VARIABLE DE EMPLEO}

El escenario del programa con referencia al empleo consiste en una visión al 2020 de 109,725 en el sector secundario, por medio del aumento en el uso urbano industrial pasando de 385.45 hectáreas (H. Ayuntamiento de Huehuetoca, 2007) a 861 en el 2020. No obstante, de acuerdo con el primer informe de gobierno de la administración municipal de Huehuetoca 20132015, para el 2013 sólo se generaron 2,780 empleos ${ }^{13}$. Sin embargo, se incorporan en estructura programática el rescate a los proyectos de desarrollo industrial que propone el programa Ciudades del Bicentenario; adicionalmente, la Comisión Coordinadora para el impulso a la Competitividad en el Estado de México, anunció la construcción de parques industriales de alta tecnología para el municipio a través del fideicomiso de desarrollo para empresas privadas; aunque no se estableció una fecha precisa para esta acción.

El crecimiento industrial que marca el programa Ciudades del Bicentenario está estrechamente relacionado con la utilización de las vías férreas que se pretendía fueran empleadas para el tren suburbano que garantizaría la movilidad de la población; no obstante, este proyecto fue interrumpido en el 2010 y es hasta el 2014 cuando ha sido retomado por parte de la Secretaría de Comunicaciones y Transportes del Gobierno Federal; el proyecto señala que el tren tendrá como destino final la ciudad de Querétaro, partiendo de la estación de Buenavista en la ciudad de México, por la misma vía que utiliza el Ferrocarril Suburbano (que llega a Cuautitlán) hasta Huehuetoca y posteriormente se construirán vías nuevas dobles para llegar a Querétaro (De la Rosa, 2014).

De acuerdo con De la Rosa (2014), se hará uso de los derechos de vía de las empresas ferroviarias de carga que contempla el trazo. Sin embargo, no se deja en claro quién será el encargado de los trabajos de ampliación del Ferrocarril Suburbano hasta Huehuetoca, lo que incide en el óptimo desarrollo del proyecto.

Derivado de la demanda de la población para transitar de México a Querétaro, el gobierno federal analiza la posibilidad de la construcción de una nueva estación de autobuses en la zona intermedia de Huehuetoca, con una inversión planteada de seis millones de pesos (De la Rosa, 2014). De llevarse a cabo estos proyectos es posible inferir que la situación de abandono en la

\footnotetext{
${ }^{13} \mathrm{El}$ informe no específica el sector al que pertenecen los empleos.
} 
vivienda que actualmente prevalece en los conjuntos urbanos disminuiría paulatinamente. Sin embargo, los incrementos poblacionales demandarían mayores requerimientos tanto de suelo como de agua potable, vialidad y servicios, aspectos que condicionan, el desarrollo urbano del municipio, cuestionando las capacidades de gestión y coordinación entre los ámbitos de gobierno estatal y local.

\section{EFECTOS DEL PROGRAMA CIUDADES DEL BICENTENARIO EN LA OCUPACIÓN DEL SUELO E INFRAESTRUCTURA HIDRÁULICA.}

Es importante mencionar en este apartado, que el último registro sobre la distribución de usos de suelo en el contexto municipal se realizó en el 2007, por su parte INEGI realizó una publicación sobre la superficie de área urbana estimada para el año 2010, en donde se aprecia que la superficie destinada al uso urbano aumentó en razón de 0.5\% del 2007 a 19,32 hectáreas en el 2010. La transposición de los mapas de usos de suelo y distribución de localidades se obtiene que los conjuntos urbanos de Santa Teresa y Urbi Villas del Rey, si bien se ubican en uso de suelo urbano se han extendido hacia áreas de pastizal; mientras que los conjuntos urbanos de El Dorado Huehuetoca, Ciudad Integral Guadalupe la Guiñada, Huehuetoca del Maurel, Galaxia Huehuetoca y Privadas del Valle, se localizan en zonas destinadas al uso agrícola (Ver figura 3). 
Figura 3. Transposición de usos de suelo y distribución de localidades

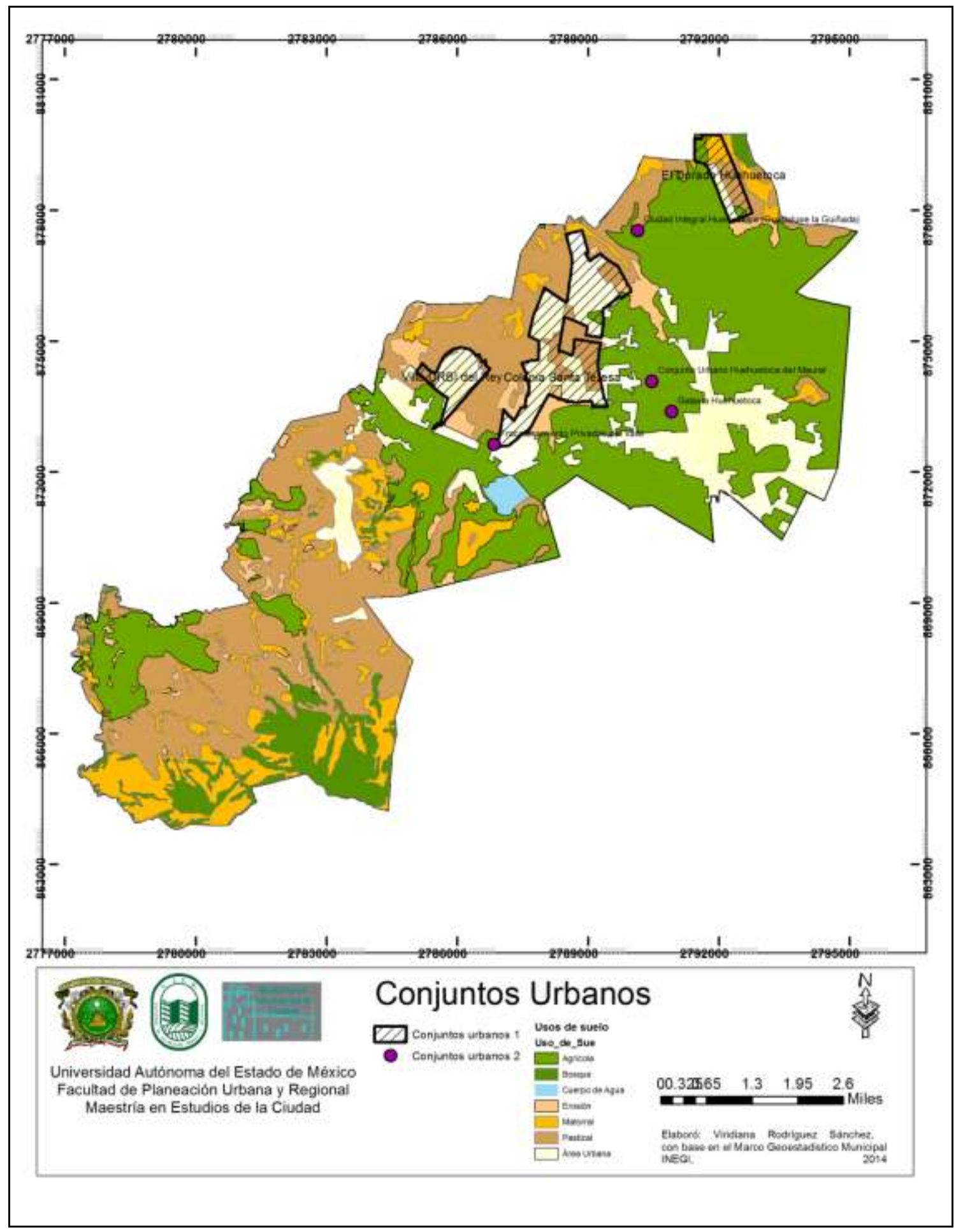

Fuente: Elaboración propia, con base en INEGI, 2013. 


\section{INFRAESTRUCTURA HIDRÁULICA}

Entre los alcances generales del programa Ciudades del Bicentenario, se plantea el contar con infraestructura de cabecera para recibir grandes contingentes de población; sin embargo, para el caso de Huehuetoca se tiene un importante déficit en materia de agua potable. Actualmente, la capacidad total de abastecimiento de agua es de 816 litros por segundo; mientras que la capacidad de funcionamiento es de 630 litros por segundo, registrando un déficit de 186 litros por segundo (H. Ayuntamiento de Huehuetoca, 2013).

De acuerdo con la administración municipal de Huehuetoca, para el año 2013 se han rehabilitado los pozos existentes en el municipio, además de prestar el servicio de agua potable a través de pipas, con beneficios para cerca de 40,000 habitantes. Adicionalmente, se plantea la perforación de un pozo profundo en el barrio de San Miguel Jagüeyes. Con referencia a las acciones planeadas por el programa Ciudades del Bicentenario, hasta el año 2014 no ha existido una coordinación entre los tres ámbitos de gobierno para el saneamiento del Río Cuautitlán, ni para ofrecer alternativas de solución a la problemática de agua potable que afecta la dotación de este servicio en los conjuntos urbanos; por su parte los actores inmobiliarios tampoco dan alternativas de solución a este problema.

La comparación de los escenarios y alcances del programa Ciudades del Bicentenario, a seis años de su implementación, permite apreciar que los resultados obtenidos hacen clara la necesidad de un reajuste en el programa contemplando los efectos colaterales que el comportamiento demográfico y la expansión de la vivienda han generado en la ocupación del suelo y suministro de agua potable. Las inconsistencias del programa, muestran la clara necesidad de la integración de los actores públicos, privados y sociales no solo en la implementación y evaluación del programa más bien en la fase de diseño desde la cual están excluidos. De existir esta visión multinivel el programa Ciudades del Bicentenario, podría ajustarse a la realidad que enfrenta hoy en día el municipio.

\section{CONCLUSIONES}

La acelerada producción de vivienda en el Estado de México sin tomar en cuenta la disponibilidad de infraestructura productiva incide en la problemática del mercado inmobiliario de vivienda, de acuerdo con INFONAVIT (2012) se registran 11,300 unidades abandonadas, lo que lo coloca en la cuarta entidad después de Tamaulipas, Chihuahua y Nuevo León; la política habitacional en la entidad durante la administración 2005-2011 privilegiaba el modelo de ciudad 
dispersa y la conformación de polos de desarrollo, a través de las Ciudades Bicentenario. No obstante, para el caso de Huehuetoca al igual que los municipios de Tecámac y Zumpango, el programa justificó el acelerado crecimiento de la vivienda vía conjuntos urbanos sin tomar en cuenta las carencia en infraestructura, servicios básicos, vialidad y equipamiento de cada municipio.

El programa Ciudades Bicentenario demostró ser una iniciativa vertical implementada desde el ámbito estatal y empresarial sin un acercamiento con el gobierno local ni con la sociedad civil que coadyuvara al análisis de la factibilidad de un proyecto de esta magnitud; a seis años de la implementación de este programa el $47.66 \%$ de la vivienda en el municipio se encuentra abandonada, ante esta situación la Secretaría de Desarrollo Urbano continua autorizando la construcción de más viviendas sin control por parte del gobierno local, factor que enfrenta al mercado inmobiliario a la disyuntiva de una venta por debajo del costo de producción o a una espera prolongada para poder colocar las viviendas en esquemas crediticios de largo plazo.

Por su parte el Gobierno Federal, en su Programa Nacional de Desarrollo Urbano y Vivienda para el periodo 2013-2018, establece como nuevo modelo de desarrollo urbano y ordenamiento territorial "la configuración de ciudades compactas y habitables con opciones de vivienda bien localizada y adecuadas a los ingresos de la población" (SEDATU, 2013: 13); modelo que consiste en un patrón completamente opuesto al planteado en las Ciudades Bicentenario.

Este nuevo modelo de crecimiento urbano da la pauta para algunas interrogantes, si la nueva administración busca la formación de ciudades compactas, ¿por qué la Secretaría de Desarrollo Urbano continua con la autorización de viviendas en la Ciudad Bicentenario Huehuetoca? Si además el $47.66 \%$ de éstas se encuentran abandonadas; ¿cuál es la estrategia para remediar esta situación?

Por su parte, el H. Ayuntamiento de Huehuetoca en su administración 2013-2015, busca continuar con los proyectos de equipamiento e infraestructura que establece el programa Ciudades Bicentenario; el sector empresarial a través del Fideicomiso de Desarrollo para Empresas Privadas planea la construcción de otro parque industrial en el municipio de Huehuetoca, tratando así de cumplir con los lineamientos del programa Ciudades Bicentenario.

Es innegable que el mercado inmobiliario de la vivienda en la zona norte del Estado de México y en particular en el municipio de Huehuetoca enfrenta retos de singular importancia, la mayoría de ellos radica en la capacidad de los tres órdenes de gobierno para concretar los proyectos estratégicos de desarrollo regional considerados como parte de las políticas de desarrollo urbano, así como del involucramiento de la iniciativa privada y de la sociedad civil, así 
como de la conciliación de intereses a fin de acercarse a esquemas de gobernanza urbana integrada como los originalmente planteados.

\section{FUENTES CONSULTADAS}

AGUILAR, Israel: Nueva Gestión Pública en México: la experiencia del Instituto del Fondo Nacional de Vivienda para los Trabajadores (2010) en: http: //sheffield.academia.edu/IsraelAguilarM\%C3\%A1rquez, 02 de marzo del 2014.

Comisión Coordinadora para el Impulso a la Competitividad del Estado de México, Programa de Desarrollo Urbano Ciudades del Bicentenario. 2007

CONAPO, Consejo Nacional de Población: Proyecciones de población al 2030 por entidad y localidad. 2014

DE LA ROSA, Alberto: Publica SCT proyecto del tren México-Querétaro en: Diario el Economista, 2014. Disponible en: http://eleconomista.com.mx/industrias/2014/04/06/publica-sct-proyectotren-mexico-queretaro.

Dirección General de Planeación Urbana de la Secretaría de Desarrollo Urbano: Ciudades del Bicentenario en: Revista de la Agencia para la Planeación del Desarrollo Urbano de Nuevo León, 3 (9), 2-6. 2008.

Fragoso Becerril, Francisco J: Vivienda en la ciudad en El Financiero, Espacio Urbano. Año 1, No. 8. Noviembre 10, 2014.

GEM, Gobierno del Estado de México: Plan Parcial de Desarrollo Urbano de Santa Teresa, (11 de marzo del 2002).

Plan Regional de Desarrollo Urbano del Valle Cuautitlán-Texcoco, (Junio, 2005).

Programa Estatal de Desarrollo Urbano, (Mayo, 2008).

Ciudades del Bicentenario rumbo a un futuro urbanamente sustentable. En Revista Habitar, 1 (2), 8-24. 2008 b.

H. Ayuntamiento de Constitucional de Huehuetoca: Modificación Parcial al Plan de Desarrollo Urbano de Huehuetoca, 2005.

Modificación del Plan de Desarrollo Urbano de Huehuetoca, 2007.

Plan Municipal de Desarrollo, 2013-2015.

INEGI, Instituto Nacional de Geografía e Informática: ITER Integración Territorial del Conteo de Población y Vivienda por Localidad, Estado de México, INEGI, 2010.

JGDF, Jefatura de Gobierno del Distrito Federal: Bando número dos, publicado en el periódico oficial del gobierno del Distrito Federal el 7 de diciembre, 2000.

INFONAVIT, Instituto Nacional del Fondo para la Vivienda de los Trabajadores, 2012: Vivienda abandonada, (mayo 2012). 
PEDROTTI, Carolina: Diseño y sustentabilidad, reflexiones en torno a la producción de conjuntos urbanos y su impacto territorial en el Estado de México en Coloquio Internacional de Diseño 2013, disponible en: http://www.2013.coloquiodediseno.org/coloquio-2010/mesa3/8.swf

RODRÍGUEZ, Isabel: Vivienda social latinoamericana: la clonación del paisaje de la exclusión en: Revista Arquitectura, Ciudad y entorno; Barcelona-España, No.2. 2013.

SCHTEIGART, Martha y GARCÍA, Beatriz: Nuevas políticas de vivienda en México. Nuevas relaciones entre sector público y privado y retos para el futuro en: La vivienda en México construyendo análisis y propuestas, Centro de Estudios Sociales y de Opinión Legal Pública, Cámara de Diputados LIX Legislatura, 2006.

SEDU, Secretaría de Desarrollo Urbano: Ciudades del Bicentenario: Una propuesta de ordenamiento del territorio para tener mejores ciudades en: 9o Reunión Nacional de Comisiones Consultivas Regionales del INFONAVIT; Metepec, México: Gobierno del Estado de México. 2008.

Estadísticas sobre conjuntos urbanos en el Estado de México 2000-2014 disponible en: http: //portal2.edomex.gob.mx/sedur/informacion_de_interes/autoriaciones/index.htm, 14 de abril del 2014.

SEDATU. Secretaría de Desarrollo Agropecuario Territorial y Urbano (2013): Programa Nacional de Desarrollo Urbano y Vivienda 2013-2018.

Universidad Autónoma del Estado de México: Estudio de Factibilidad de la Unidad Académica Profesional Huehuetoca, Secretaría de Docencia. Dirección de Estudios Profesionales, 2012.

Trabalho enviado em 06 de fevereiro de 2015.

Aceito em 11 de fevereiro de 2015. 